\title{
Redução da dose de radiação em tomografia computadorizada em crianças com traumatismo cranioencefálico não compromete o diagnóstico e a conduta
}

\author{
Reduction of radiation dose of computed tomography in children with traumatic brain \\ injury does not compromise the diagnosis and medical conduct
}

\begin{abstract}
RESUMO
Introdução: $O$ aumento na disponibilidade e solicitação de tomografias computadorizadas para crianças traz preocupação pelo efeito deletério e cumulativo da radiação. O traumatismo cranioencefálico é uma situação em que a tomografia é frequentemente necessária. Objetivos: 1. Avaliar se a redução da dose de radiação da tomografia computadorizada de crânio em crianças com traumatismos cranioencefálicos prejudica o diagnóstico e a conduta; 2 . Promover campanha de radioproteção em um serviço de saúde suplementar. Método: Selecionamos dois grupos de tomografias computadorizadas de crânio de crianças com traumatismo cranioencefálico: 30 previamente realizadas com doses de radiação habitual; e 30 realizadas durante o projeto, nas quais foi aplicado o protocolo The Alliance for Radiation Safety in Pediatric Imaging com redução de aproximadamente 50\% da dose de radiação. As duas séries de exames foram apresentadas a 19 pediatras, 2 neurocirurgiões e 7 radiologistas que desconheciam as diferenças técnicas e responderam a um questionário. Resultados: Os profissionais não tiveram dificuldade em realizar o diagnóstico e estabelecer conduta com ambas as séries de exames. Quatro participantes observaram maior granulação das imagens nos exames com menor dose de radiação. Foi criada a campanha de radioproteção com distribuição de 17 mil carteiras de radioproteção para crianças até 12 anos. Os profissionais envolvidos e os pais aderiram forte e racionalmente à campanha. Conclusões: Foi possível reduzir a dose de radiação das tomografias computadorizadas em crianças com traumatismo cranioencefálico sem prejuízo ao diagnóstico e à conduta. A campanha de radioproteção foi efetiva e bem aceita pelos profissionais e familiares e irá se transformar em campanha nacional.
\end{abstract}

Palavras-chave: proteção radiológica; educação em saúde; tomografia; anormalidades induzidas por radiação; traumatismos cranioencefálicos.

\begin{abstract}
Introduction: The worldwide increases in availability and request of computed tomography in children have brought concern about the cumulative effect of radiation. Traumatic brain injury is a clinical situation in which tomography is frequently necessary. Objectives: 1. Evaluating if the reduction of the radiation dose of head computed tomography in children with head trauma would affect the diagnosis and medical conduct; 2 . Promoting a radioprotection campaign in a private health care system. Method: We selected two groups of computed tomography from children with head trauma: 30 performed before the study period, with usual doses of radiation; and 30 tomographies performed during the project, in which we applied the protocol of The Radiation Safety The Alliance for Imaging in Pediatric with 50\% radiation dose reduction. The two series of exams were presented to 19 pediatricians, 2 neurosurgeons and 7 radiologists who were unaware of the technical differences and they answered a questionnaire. Results: The professionals had no difficulty in making a diagnosis and establish a conduct with both series of exams. Four participants noted more grainy images in the exams with lower radiation dose. A radioprotection campaign distributed 17,000 radioprotection wallets for children up to 12 years. Professionals involved and parents joined the campaign strongly and rationally. Conclusions: It is possible to reduce the computed tomography radiation dose for children with head trauma without any prejudice to the diagnosis and treatment. The radioprotection campaign was effective and well accepted by professionals and family and will become a national campaign.

Keywords: radiation protection; health education; tomography; abnormalities, radiation-induced; head trauma.
\end{abstract}

${ }^{1}$ Pontifícia Universidade Católica de São Paulo (PUC-SP), Faculdade de Ciências Médicas e da Saúde - Sorocaba (SP), Brasil. Contato: mo.bernardo@yahoo.com.br

Recebido em 20/09/2016. Aceito para publicação em 27/11/2016. 


\section{INTRODUÇÃO}

O aumento na solicitação e a facilidade na execução de exames radiológicos, em particular a tomografia computadorizada (TC), realizada principalmente em equipamentos helicoidais e MultiSlice, têm trazido preocupação no meio médico e científico devido ao efeito cumulativo da dose de radiação. Nos Estados Unidos, o número estimado de tomografias por ano era de 3 milhões na década de 1980, aumentando para 62 milhões em 2007. ${ }^{1}$ De acordo com o último relatório da United Nations Scientific Committee on the Effects of Atomic Radiation (UNSCEAR), de 3 a $10 \%$ de todos os procedimentos médicos por imagem são realizados em crianças. ${ }^{2,3}$

A TC, que contribuía com cerca de 2 a $3 \%$ de todos os exames radiológicos, passou a $30 \%$ em virtude do aumento do seu espectro de uso, da facilidade e disponibilidade dos equipamentos em centros ambulatoriais e hospitalares. ${ }^{4}$ No Reino Unido, a TC contribui com $50 \%$ da dose emitida de todos os exames radiológicos. ${ }^{5}$

As crianças e os adolescentes, devido ao fato de seus tecidos ainda estarem em desenvolvimento, possuem maior sensibilidade e risco para os efeitos da radiação ionizante, sendo tanto maior quanto mais jovem for a criança. ${ }^{3,6}$

Há muitos estudos indicando o aumento do risco de crianças expostas à $\mathrm{TC}$, em relação às não expostas, desenvolverem doenças degenerativas, particularmente o câncer. Um estudo americano estimou o aumento do risco de mortalidade por câncer que uma criança de 1 ano de idade tem após ser exposta à radiação de uma TC de abdome $(0,18 \%)$ ou TC de crânio $(0,07 \%)$, evidenciando que, embora o risco seja baixo, ele não é desprezível. ${ }^{7}$ Esse estudo estima que, no final da década de 1990, nos EUA, eram realizadas 600 mil TC de abdome ou de crânio anuais em crianças com menos de 15 anos. Além disso, o estudo infere, grosseiramente, que 500 desses indivíduos irão morrer de câncer, o que pode ser atribuído ao exame. ${ }^{7}$

Segundo o Life Span Study, os efeitos da baixa dose acumulativa da radiação ionizante da TC - predominantes nos indivíduos do sexo feminino e mais jovens - podem ser comparáveis, proporcionalmente, aos observados nos sobreviventes das bombas atômicas no Japão. Sobreviventes da bomba de Hiroshima desenvolveram câncer com doses de radiação correspondentes a duas ou três TC de abdome em um adulto. ${ }^{8-12}$

No Reino Unido, 180 mil jovens que fizeram tomografias de baixa dose foram acompanhados de 1985 a 2002 e os resultados indicaram aumento da incidência de leucemia e tumor cerebral. ${ }^{13} \mathrm{O}$ estudo também revelou que a realização de cinco a dez TC de crânio em crianças abaixo de 15 anos resultava em dose cumulativa de radiação na medula óssea, o que triplica o risco de leucemia. Da mesma forma, a realização de duas a três TC de crânio também triplica o risco de câncer cerebral. ${ }^{13}$

Um estudo recém-publicado, que acompanhou $10 \mathrm{mi}$ lhões de crianças e adolescentes na Austrália, evidenciou aumento de $24 \%$ de risco de câncer na população exposta à TC, em relação à não exposta, sendo esse aumento tanto maior quanto mais jovem fosse a criança e proporcional ao núme- ro de exames realizados. ${ }^{6} \mathrm{O}$ excesso absoluto da incidência de câncer nas crianças expostas em um acompanhamento de 9,5 anos foi de 9,38 por 100 mil indivíduos, com predomínio para os tumores de órgãos sólidos e tecido linfoide. ${ }^{6}$

Habitualmente, os indivíduos são expostos a uma radiação natural (underground radiation). Uma TC de crânio pode acumular em radiação o equivalente a oito meses de exposição natural. ${ }^{14}$

O Colégio Americano de Radiologia e a Sociedade Americana de Pediatria, preocupados com o aumento de TC realizadas e o efeito somatório da dose de radiação absorvida, criaram a Alliance for Radiation Safety in Pediatric Imaging 2007 - The Image Gently Campaign. ${ }^{15}$

Para minimizar os riscos, foram propostos protocolos de redução de dose em exames radiológicos. Além disso, os especialistas recomendam: utilizar protocolos com doses reduzidas segundo parâmetros adequados à idade e ao peso da criança; realizar o exame direcionado à área a ser estudada; evitar o uso de múltiplas fases e sequências durante o exame; e, quando possível, considerar o uso de outros métodos alternativos, tais como ultrassom e ressonância magnética nuclear. ${ }^{14}$

Instituições afiliadas à Agencia Internacional de Energia Atômica e Unidade de Radioproteção ao paciente irão monitorar a redução de $52 \%$ na dose de radiação, já obtida entre 2008 e 2010 em TC de crânio. ${ }^{16}$ Membros do mesmo grupo desenvolveram um cartão eletrônico de dosagem de radiação que funciona como histórico do paciente. ${ }^{2}$

A preocupação com o monitoramento de dose de radiação em jovens nos motivou a desenvolver um projeto com crianças com traumatismo cranioencefálico (TCE) atendidas na unidade de emergência de um hospital próprio de uma cooperativa médica de uma cidade de grande porte no interior do estado de São Paulo. Escolhemos a TC de crânio em crianças, pois é o exame mais utilizado na avaliação do TCE. No Brasil, tem-se observado aumento da taxa de TCE em crianças menores de 10 anos. ${ }^{17} \mathrm{Em}$ unidades de urgência e emergência, a TC de crânio é indispensável para realizar o diagnóstico precoce, orientar a conduta e reduzir o tempo de permanência dos pacientes no ambiente hospitalar. ${ }^{18,19}$

Assim, o objetivo do presente estudo foi avaliar se a redução da dose de radiação utilizada em TC de crânio prejudica a interpretação do exame, o diagnóstico ou a conduta em crianças com TCE. Intencionava-se também desencadear, no ambiente de trabalho, a discussão e a implementação de medidas capazes de reduzir a dose de radiação recebida por crianças que necessitam de atenção à saúde e estimular a conscientização dos pediatras e dos familiares quanto aos perigos da radiação excessiva.

\section{METODOLOGIA}

Trata-se de uma pesquisa de abordagem quantitativa, em relação à técnica do exame radiológico, e de natureza qualitativa, na avaliação da opinião dos especialistas em radiologia e dos médicos envolvidos no atendimento à criança com TCE.

\section{Seleção do material radiológico}

Criamos, a partir do registro do Serviço de Radiologia do hospital, um banco de dados com TC de crânio de crianças 
que sofreram TCE. No grupo "controle" (que também chamamos de grupo com "dose habitual de radiação"), foram incluídos 30 exames: realizados nas últimas crianças com idade de 0 a 10 anos atendidas na unidade de urgência e emergência do hospital, no período de janeiro a agosto de 2012. Caracterizamos o grupo e quantificamos as doses de radiação aplicada a essa série de exames.

Posteriormente, na execução dos exames nos 30 atendimentos seguintes de crianças com TCE (nos quais foram solicitadas TC de crânio, garantindo a adequação da técnica radiológica), adotamos o protocolo de redução de dose de radiação, limitada a $150 \mathrm{mAs}$, seguindo as orientações da The Alliance for Radiation Safety in Pediatric Imaging da Sociedade Americana de Pediatria e do Colégio Americano de Radiologia, que levam em conta as dimensões, o peso e a idade das crianças. ${ }^{14,15}$ Utilizamos a menor dosagem de quilo voltagem (KV) que o equipamento de tomografia sugeriu e limitamos o comprimento e a extensão do campo de exposição somente à área necessária referida na solicitação médica. Esses 30 novos exames constituíram o grupo "dose reduzida de radiação". Ao computarmos a dose total (DLP) emitida, referida pelo equipamento de tomografia nos exames desses pacientes, identificamos uma redução aproximada de 50\% da dose de radiação (ver resultados). Em nenhuma das séries, houve necessidade de repetir o exame.

O número de exames em cada grupo foi determinado por conveniência e em quantidade suficiente para garantir um amplo espectro de alterações radiológicas comuns em casos de TCE, especificamente: hematoma subgaleal, hematoma parenquimatoso, hematoma extra-axial e fratura. Houve semelhança de diagnósticos entre os dois grupos estudados (ver resultados).

\section{Participantes da pesquisa}

Em posse das duas séries de exames, analisamos se, na opinião dos radiologistas e dos médicos envolvidos no atendimento às crianças com TCE, havia diferença entre as duas séries de exames e se essas diferenças poderiam alterar o diagnóstico radiológico ou a conduta terapêutica. Os médicos analisaram as imagens por meio do sistema de visualização do hospital, utilizando o mesmo computador e o monitor onde habitualmente avaliam os exames e tendo apenas o número do exame como identificação do paciente. Todos os médicos envolvidos no atendimento de crianças com TCE, presentes no ambiente de trabalho e no período em que a pesquisa foi realizada, foram convidados a participar. Aceitaram participar da pesquisa 7 radiologistas (6 homens, idade média de 48 anos), 19 pediatras (13 mulheres e idade média de 51 anos) e 2 neurocirurgiões ( 2 homens, com idade média de 52 anos).

\section{Aplicação do questionário e avaliação dos exames pelos participantes da pesquisa}

Os participantes receberam duas séries de TC de crânio, sendo que, em cada série havia três exames com "dose habitual de radiação" e três exames com "dose reduzida de radiação". Incluímos sempre um exame normal e dois exames com as alterações radiológicas mais comuns (ver Tabelas 1 e 2). Após avaliar as duas séries de exames, os participantes responderam um questionário estruturado que tinha por objetivo identificar: 1) se reconheciam alguma diferença entre as duas séries de exames; e 2) se a qualidade técnica dos exames permitia o diagnóstico e a conduta adequada em cada caso. Além disso, o questionário abordava se o participante via a necessidade de capacitação técnica e se considerava útil a criação de uma carteira de radioproteção na qual seriam registrados os exames radiológicos da criança com o objetivo de conscientizar familiares e profissionais de saúde no sentido de reduzir a carga de radiação à qual as crianças estão expostas.

\section{Aspectos éticos}

O protocolo do estudo e o Termo de Consentimento Livre e Esclarecido (TCLE) para os médicos participantes foram aprovados pelo Comitê de Ética em Pesquisa da Faculdade de Ciências Médicas e da Saúde da Pontifícia Universidade Católica de São Paulo, campus Sorocaba. Entendemos que seria desnecessário o TCLE para os pacientes e responsáveis, pois os exames da série de "dose habitual de radiação" já tinham sido realizados dentro da rotina própria do serviço, e, da mesma forma, os exames com "dose reduzida de radiação" proposta para os procedimentos radiológicos realizados a partir do início do projeto foram feitos de acordo com as recomendações das diretrizes do Colégio Americano de Radiologia e da Sociedade Americana de Pediatria. Os exames não identificavam a criança e os dados foram tratados com absoluto sigilo pelos pesquisadores, como expresso no Termo de Confidencialidade.

\section{RESULTADOS}

A Tabela 1 apresenta a identificação numérica, os parâmetros demográficos, os diagnósticos radiológicos e as doses correspondentes a cada exame realizado na série de "dose habitual de radiação". Dos 30 exames selecionados, 14 foram considerados normais e 16 tinham alterações consequentes ao TCE ou preexistentes. Seis exames apresentavam mais de uma alteração ou diagnóstico radiológico.

A Tabela 2 apresenta as mesmas informações, agora para a série de exames realizados com "dose reduzida de radiação". Observe que a distribuição etária, de gênero e os diagnósticos radiológicos nos dois grupos de crianças são semelhantes. A média das doses de radiação (DLP) na série de TC realizada com doses habituais (Tabela 1) foi de 1082,8 mGy.cm e da série de TC com doses reduzidas (Tabela 2) foi de 537,1 mGy. $\mathrm{cm}$, correspondendo à redução de aproximadamente $50 \%$ da dose total de radiação nos exames da última série ( $\mathrm{p}<0,0001$, teste $t$ de Student).

A Figura 1 mostra exemplos de TC de crânio com doses habituais e com doses reduzidas de radiação de crianças com TCE. Em dois casos, as setas apontam hematomas extra-axiais e em dois casos apontam fraturas ósseas. As doses de radiação estão expressas em cada uma das imagens apresentadas. 
Análise e opinião dos médicos participantes da pesquisa

A análise dos exames pelos médicos responsáveis pelos cuidados às crianças com TCE mostrou que um neurocirurgião, dois radiologistas e um pediatra afirmaram ter observado maior "ruído" (granulação da imagem) nos exames com redução da carga de radiação. Apesar de esses profissionais identificarem essa pequena diferença nas duas séries de exames, nenhum deles teve dificuldade em realizar o diagnóstico e orientar a conduta com base nas duas séries de exames com "dose habitual" ou "dose reduzida" de radiação. Os demais participantes não identificam diferenças entre as duas séries de exames nem tiveram dificuldades diagnósticas ou de conduta.

Com exceção de um pediatra e um radiologista, todos os demais responderam que desejavam participar de um pro-

Tabela 1. Características das tomografias computadorizadas de crânio de crianças com traumatismo cranioencefálico, realizadas com "dose habitual de radiação".

\begin{tabular}{|c|c|c|c|c|c|}
\hline $\begin{array}{l}\text { Registro } \\
\text { do paciente }\end{array}$ & $\begin{array}{c}\text { Sexo } \\
\text { Mas./Fem. }\end{array}$ & $\begin{array}{c}\text { Idade } \\
\text { (meses) }\end{array}$ & $\begin{array}{l}\text { Diagnóstico } \\
\text { radiológico }^{1}\end{array}$ & $\mathbf{m A s} \mathbf{s}^{2}$ & $\begin{array}{c}\text { DLP }^{3} \\
(\mathrm{mGy} \cdot \mathrm{cm})\end{array}$ \\
\hline 502378 & M & 95 & $\mathrm{FC} / \mathrm{S}$ & 349 & 1323 \\
\hline 339930 & M & 57 & $\mathrm{FC}$ & 251 & 873 \\
\hline 453420 & M & 23 & $\mathrm{FC}$ & 349 & 1023 \\
\hline 446628 & $\mathrm{~F}$ & 27 & FC/HEA/HSG & 349 & 938 \\
\hline 407450 & M & 44 & $\mathrm{FC} / \mathrm{S}$ & 398 & 1178 \\
\hline 411231 & M & 88 & FC/HSG/FO & 300 & 842 \\
\hline 363610 & M & 83 & HSG/FN & 300 & 819 \\
\hline 341819 & M & 80 & HSG & 398 & 1209 \\
\hline 452776 & M & 1 & HP & 349 & 833 \\
\hline 343055 & M & 25 & HSG & 398 & 1111 \\
\hline 351402 & $\mathrm{~F}$ & 25 & HSG & 398 & 976 \\
\hline 368315 & M & 50 & HSG & 398 & 1147 \\
\hline 456865 & $\mathrm{~F}$ & 68 & HSG & 349 & 944 \\
\hline 390807 & $\mathrm{~F}$ & 83 & HSG & 300 & 884 \\
\hline 386458 & F & 61 & HEA/S & 398 & 1333 \\
\hline 341763 & $\mathrm{~F}$ & 96 & $\mathrm{~N}$ & 398 & 1125 \\
\hline 343483 & F & 103 & $\mathrm{~N}$ & 398 & 1221 \\
\hline 345242 & F & 19 & $\mathrm{~N}$ & 398 & 1184 \\
\hline 348782 & F & 30 & $\mathrm{~N}$ & 398 & 1178 \\
\hline 350794 & F & 81 & $\mathrm{~N}$ & 398 & 1059 \\
\hline 354510 & M & 40 & $\mathrm{~N}$ & 398 & 1118 \\
\hline 404080 & $\mathrm{~F}$ & 91 & $\mathrm{~N}$ & 398 & 1127 \\
\hline 355623 & M & 9 & $\mathrm{~N}$ & 398 & 1098 \\
\hline 362158 & $\mathrm{~F}$ & 77 & $\mathrm{~N}$ & 398 & 1131 \\
\hline 365338 & M & 40 & $\mathrm{~N}$ & 398 & 1152 \\
\hline 366804 & M & 75 & HSG & 398 & 1168 \\
\hline 366884 & M & 130 & $\mathrm{~N}$ & 398 & 1168 \\
\hline 359736 & F & 92 & $\mathrm{~N}$ & 398 & 1147 \\
\hline 445655 & M & 114 & $\mathrm{~N}$ & 349 & 1056 \\
\hline 431791 & M & 95 & $\mathrm{~N}$ & 398 & 1116 \\
\hline Média & $17 \mathrm{M} / 13 \mathrm{~F}$ & 63,4 & 14N/16 Alt. & & 1082,7 \\
\hline DP & & 33,8 & & & 137,8 \\
\hline
\end{tabular}

1. Diagnóstico radiológico: $\mathrm{N}=$ exame normal; $\mathrm{HP}=$ hematoma parenquimatoso; HSG=hematoma subgaleal; HEA=hematoma extra-axial; $\mathrm{FC}=$ fratura da calota craniana; $\mathrm{FO}=$ fratura orbitária; $\mathrm{FN}=$ fratura do osso nasal; $\mathrm{S}=$ sinusopatia. $2 . \mathrm{mAs}=\mathrm{Miliamperes} / \mathrm{s} .3 ; \mathrm{DLP}=\mathrm{Dose}$ total. 
grama de educação continuada que inclui aspectos técnicos relacionados à solicitação, realização ou interpretação de exames radiológicos por meio de palestras, informativos pela página eletrônica do cooperado e reuniões científicas.

Com relação à carteira de radioproteção, apenas dois pediatras mencionaram que não achavam necessária a implantação de tal controle, considerando preferível a explicação verbal para as famílias e por recear que um documento dessa natureza cause preocupações excessivas os pais. Os demais consideraram importante o uso da carteira de radioproteção e mostraram-se receptivos e muito motivados com a ideia. Vários pediatras relataram a necessidade de orientação da família, pois muitos pais, desconhecendo os riscos, vêm à consulta com a ideia de que a criança necessita um exame radiológico, pressionando os médicos a solicitarem o exame. Por isso, consideram que a carteira de radioproteção seria

Tabela 2. Características das tomografias computadorizadas de crânio de crianças com traumatismo cranioencefálico, realizadas com "dose reduzida de radiação".

\begin{tabular}{|c|c|c|c|c|c|}
\hline $\begin{array}{l}\text { Registro } \\
\text { do paciente }\end{array}$ & $\begin{array}{c}\text { Sexo } \\
\text { Mas./Fem. }\end{array}$ & $\begin{array}{c}\text { Idade } \\
\text { (meses) }\end{array}$ & $\begin{array}{l}\text { Diagnóstico } \\
\text { padiológico }^{1}\end{array}$ & $\mathbf{m A s} \mathbf{s}^{2}$ & $\begin{array}{c}\text { DLP }^{3} \\
\text { mGy.cm }\end{array}$ \\
\hline 454773 & F & 107 & HEA/FC & 200 & 573 \\
\hline 381934 & M & 32 & FC/HSG/S & 200 & 544 \\
\hline 416969 & M & 2 & HEA/FC & 200 & 446 \\
\hline 357309 & $\mathrm{~F}$ & 24 & HSG & 251 & 679 \\
\hline 342434 & $\mathrm{~F}$ & 29 & HSG & 251 & 679 \\
\hline 369931 & $\mathrm{~F}$ & 25 & HSG & 150 & 455 \\
\hline 382192 & $\mathrm{~F}$ & 40 & HSG/FC & 398 & 641 \\
\hline 409319 & M & 30 & HSG & 200 & 556 \\
\hline 357312 & $\mathrm{~F}$ & 36 & HSG & 251 & 621 \\
\hline 365705 & M & 75 & $\mathrm{~N}$ & 200 & 583 \\
\hline 350639 & $\mathrm{~F}$ & 10 & $\mathrm{~N}$ & 200 & 541 \\
\hline 444779 & $\mathrm{~F}$ & 6 & $\mathrm{~N}$ & 200 & 469 \\
\hline 419404 & M & 52 & $\mathrm{~N}$ & 200 & 557 \\
\hline 416655 & $\mathrm{~F}$ & 7 & $\mathrm{~N}$ & 200 & 590 \\
\hline 416546 & M & 2 & $\mathrm{~N}$ & 251 & 644 \\
\hline 387461 & M & 32 & HSG/FC & 200 & 557 \\
\hline 387717 & $\mathrm{~F}$ & 108 & $\mathrm{~N} / \mathrm{S}$ & 200 & 531 \\
\hline 387854 & $\mathrm{~F}$ & 67 & $\mathrm{~N}$ & 200 & 567 \\
\hline 381397 & M & 48 & $\mathrm{~N}$ & 150 & 414 \\
\hline 369961 & $\mathrm{~F}$ & 108 & $\mathrm{~N}$ & 200 & 531 \\
\hline 368577 & M & 12 & $\mathrm{~N}$ & 200 & 513 \\
\hline 365289 & $\mathrm{~F}$ & 69 & $\mathrm{~N}$ & 200 & 549 \\
\hline 427778 & M & 111 & $\mathrm{~N} / \mathrm{S}$ & 200 & 567 \\
\hline 436284 & $\mathrm{~F}$ & 54 & $\mathrm{~N}$ & 200 & 539 \\
\hline 434518 & $\mathrm{~F}$ & 49 & $\mathrm{~N}$ & 200 & 562 \\
\hline 362912 & M & 31 & $\mathrm{~N} / \mathrm{S}$ & 200 & 338 \\
\hline 360283 & $\mathrm{~F}$ & 15 & $\mathrm{~N}$ & 150 & 352 \\
\hline 357156 & M & 32 & $\mathrm{~N}$ & 200 & 510 \\
\hline 453748 & $\mathrm{~F}$ & 43 & $\mathrm{~N}$ & 200 & 544 \\
\hline 390123 & $\mathrm{~F}$ & 7 & $\mathrm{~N}$ & 300 & 462 \\
\hline Média & $13 \mathrm{M} / 17 \mathrm{~F}$ & 42,1 & 20 N/10 Alt. & & 537,1 \\
\hline DP & & 32,9 & & & 82,1 \\
\hline
\end{tabular}

1. Diagnóstico radiológico: $\mathrm{N}=$ exame normal; $\mathrm{HSG}=$ hematoma subgaleal; $\mathrm{HEA}=$ hematoma extra-axial; $\mathrm{FC}=$ fratura da calota craniana; $\mathrm{S}=$ sinusopatia. 2. $\mathrm{mAs}=$ Miliamperes/s. 3; DLP=Dose total. 
uma ferramenta importante de informação e conscientização dos pais, de modo que eles fiquem mais seguros com a atuação médica.

\section{Consequências do Desenvolvimento da Pes- quisa no Ambiente de Trabalho}

\section{Padronização dos Protocolos e Controles}

Foi realizada a capacitação de todos os profissionais técnicos envolvidos na realização de TC, de modo a instruí -los a limitar o exame exclusivamente à área de interesse descrita na solicitação; foi limitada também a emissão da dose de radiação em exames de crânio em crianças, segundo os critérios propostos no estudo, e estendida essa padronização a todos os exames radiológicos realizados no hospital. Os técnicos de radiologia envolvidos tiveram ótima aceitação do processo e sentiram-se estimulados a realizar o trabalho com as precauções necessárias, entendendo claramente o propósito da educação permanente em serviço.

\section{Implantação de Carteira de Radioproteção}

À semelhança do que foi feito em algumas campanhas internacionais, a diretoria da cooperativa desenvolveu uma carteira de radioproteção individual para crianças com idade infe-
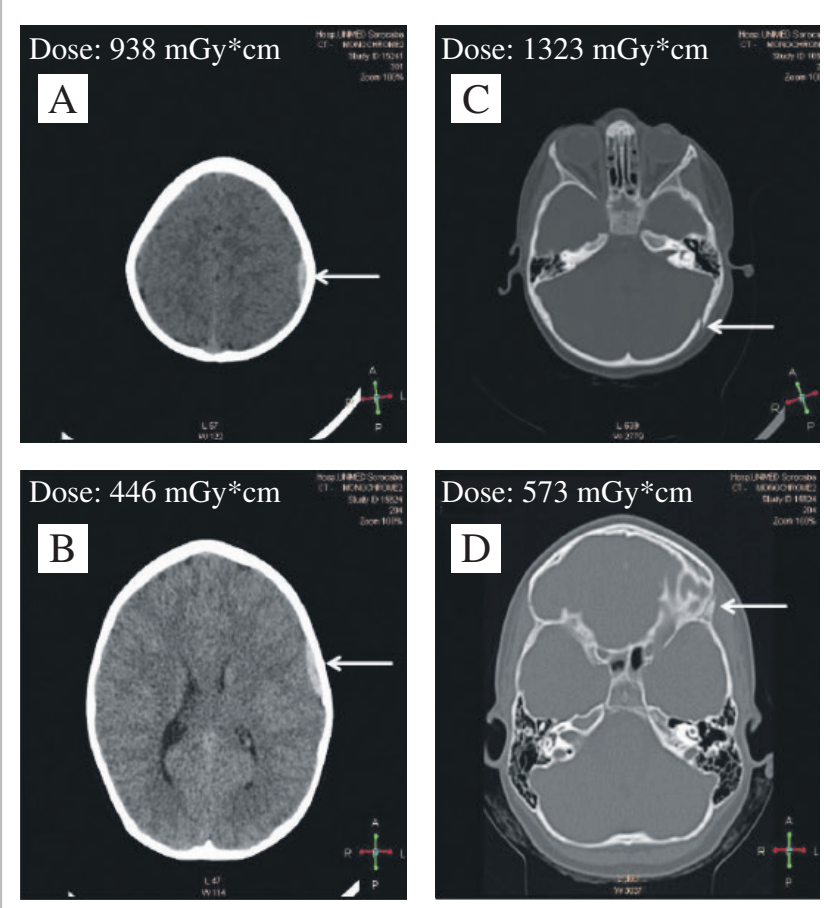

Figuras A e B correspondem a tomografias computadorizadas (TC) realizadas com dose habitual (A) e dose reduzida (B) de radiação. As setas apontam para a presença de hematomas extra-axiais. C e D correspondem a TC realizadas com dose habitual (C) e dose reduzida (D) de radiação. As setas apontam para a presença de fraturas ósseas.

Figura 1. Exemplos de tomografias computadorizadas de crânio de crianças com traumatismo cranioencefálico, realizadas com doses habituais e doses baixas de radiação. rior a 12 anos a fim de registrar todos os exames radiológicos a que sejam submetidas, o tipo de exame, as incidências e a data de realização. A carteira de radioproteção foi entregue a todas as crianças até 12 anos nos consultórios pediátricos, setores de emergência do hospital, terapia intensiva, berçário e setor de imagem. Foram distribuídas 17 mil carteiras de radioproteção. Agora, os médicos que fizerem qualquer solicitação de exames radiológicos terão em mãos um histórico imediato que poderá auxiliá-los a tomar a decisão na indicação do exame mais adequado. Para os pais, a carteira de radioproteção tem o objetivo de conscientizar sobre os efeitos de doses cumulativas de radiação.

\section{Acompanhamento das Iniciativas Motivadas} pela Realização do Estudo.

O projeto já estimulou os pediatras a buscarem a racionalização na solicitação dos exames radiológicos, os familiares a se conscientizarem quanto aos riscos da radiação e a equipe de radiologia a trabalhar com protocolos de baixa dose e radioproteção.

Um ano depois de terminado o estudo, foi realizado um levantamento aleatório de 30 TC de crânio em crianças com TCE com as mesmas caraterísticas daquelas avaliadas no estudo. Essa avaliação mostrou que a dose total de radiação (DLP) conti-

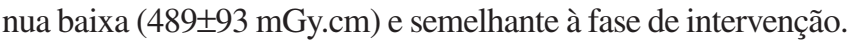

Como corolário, a direção nacional da cooperativa médica adotou a iniciativa da carteira de radioproteção, assim como todos os possíveis cuidados de redução das doses de radiação, transformando-os em um projeto nacional do sistema brasileiro da cooperativa.

\section{DISCUSSÃO}

Nosso estudo confirmou os achados da literatura no sentido de que realmente é possível reduzir as doses de radiação aplicadas a exames de TC sem perder a qualidade do exame e sem interferir no diagnóstico e na conduta dos profissionais envolvidos no cuidado com a saúde da criança. ${ }^{14,16}$

$\mathrm{O}$ projeto foi bem aceito e teve grande repercussão na comunidade, tanto entre os médicos e demais profissionais de saúde, como também na área administrativa e de qualidade do hospital, estendendo-se aos usuários e seus responsáveis. O estudo serviu de base para promoção da implantação de protocolos padronizados com o objetivo de racionalizar a dose de radiação aplicada aos exames realizados no setor de imagem do hospital. Motivou também uma campanha de radioproteção para crianças e a implantação da carteira de radioproteção dirigida às crianças de 0 a 12 anos, estimulando a conscientização dos profissionais de saúde e dos familiares quanto aos perigos e cuidados com a radiação ionizante, particularmente seu efeito cumulativo, mas sem pânico, com bom senso e cautela.

Em conclusão, este estudo mostrou que a tecnologia disponível permite reduzir as doses de radiação em crianças, protegendo-as de riscos futuros, sem interferir no processo de diagnóstico e de tratamento quando necessário. Ao mesmo tempo, promoveu intensas repercussões positivas no ambiente de trabalho e na comunidade por meio da implantação da carteira 
de radioproteção e de capacitações para toda a cadeia de atendimento, desde os profissionais diretamente envolvidos até os setores administrativos. Mostrou também que a metodologia científica, aliada às iniciativas motivacionais, pode e deve permear a prática profissional, promovendo transformações no dia a dia das instituições com benefícios para a população atendida.

\section{REFERÊNCIAS}

1. Brenner DJ, Hall EJ. Computed tomography - an increasing source of radiation exposure. N Engl J Med. 2007;357(22):2277-84.

2. Rehani MM, Kushi JF. A study of smart card for radiation exposure history of patient. AJR Am J Roentgenol. 2013;200(4):780-2.

3. United Nations Scientific Committee on the Effects of Atomic Radiation. Sources, effects and risks of ionizing radiation. New York: UNSCEAR; 2013.

4. Naik KS, Ness LM, Bowker AM, Robinson PJ. Is computed tomography of the body overused? An audit of 2068 attendances in a large acute hospital. Br J Radiol. 1996;69(818):126-31.

5. Needham G. Making the best use of a department of clinical radiology - guidelines for doctors. Health Bull. 1996;54(5):406-9.

6. Mathews JD, Forsythe AV, Brady Z, Butler MW, Goergen SK, Byrnes GB, et al. Cancer risk in 680,000 people exposed to computed tomography scans in childhood or adolescence: data linkage study of 11 million Australians. BMJ. 2013;346:f2360.

7. Brenner D, Elliston C, Hall E, Berdon W. Estimated risks of radiation-induced fatal cancer from pediatric CT. AJR Am J Roentgenol. 2001;176(2):289-96.

8. Shimizu Y, Kato H, Schull WJ. Studies of the mortality of A-bomb survivors. 9. Mortality, 1950-1985: Part 2. Cancer mortality based on the recently revised doses (DS86). Radiat Res. 1990;121(2):120-41.

9. Preston DL, Kusumi S, Tomonaga M, Izumi S, Ron E, KuramotoA, etal.Cancerincidencein atomic bombsurvivors. Part III. Leukemia, lymphoma and multiple myeloma, 19501987. Radiat Res. 1994;137(2 Suppl):S68-97.
10. Gordon SW, Schandorf C, Yeboah J. Optimization of radiation protection for the control of occupational exposure in Ghana. Radiat Prot Dosimetry. 2011;147(3):386-93.

11. Royal HD. Effects of low level radiation-what's new? Semin Nucl Med. 2008;38(5):392-402.

12. World Health Organization; International Agency for Research on Cancer. IARC Monographs on the evaluation of carcinogenic risks to humans: ionizing radiation, part I: $X$ - and gamma $(\gamma)$ - radiation, and neutrons. Lyon: IARC; 2000. v. 75.

13. Pearce MS, Salotti JA, Little MP, McHugh K, Lee C, Kim KP, et al. Radiation exposure from CT scans in childhood and subsequent risk of leukaemia and brain tumours: a retrospective cohort study. Lancet. 2012;380(9840):499-505.

14. American College of Radiology. ACR Appropriateness Criteria $^{\circledR}$ [Internet]. 2016 [Cited 2016 Dec 10] Available from: http://www.acr.org/Quality-Safety/ Appropriateness-Criteria

15. Goske MJ, Applegate KE, Boylan J, Butler PF, Callahan MJ, Coley BD, et al. The Image Gently campaign: working together to change practice. AJR Am J Roentgenol. 2008;190(2):273-4.

16. Alsuwaidi JS, Albalooshi LG, Alawadhi HM, Rahanjam A, Elhallag MA, Ibrahim JS, et al. Continuous monitoring of CT dose indexes at Dubai hospital. AJR Am J Roentgenol. 2013;201(4):858-64.

17. Koizumi MS, Lebrão ML, Mello-Jorge MH, Primerano V. [Morbidity and mortality due to traumatic brain injury in São Paulo City, Brazil, 1997]. Arq NeuroPsiquiatr. 2000;58(1):81-9.

18. Batlle JC, Hahn PF, Thrall JH, Lee SI. Patients imaged early during admission demonstrate reduced length of hospital stay: a retrospective cohort study of patients undergoing cross-sectional imaging. J Am Coll Radiol. 2010;7(4):269-76.

19. Paterson A, Frush DP. Dose reduction in paediatric MDCT: general principles. Clin Radiol. 2007;62(6):507-17. 